\title{
Patient Blood Management: die maschinelle Autotransfusion in der Anästhesiologie
}

Sabine Westphal, Kai Zacharowski, Suma Choorapoikayil,

Christoph Füllenbach, Patrick Meybohm

\section{Funktionsweise der MAT}

Alle auf dem Markt befindlichen Systeme arbeiten nach dem Zentrifugenprinzip. Nach Auffangen des Wundoder Drainageblutes darf dieses dem Patienten nicht einfach ohne Aufarbeitung zurückgegeben werden. Die Gründe dafür sind u.a. die Gefahr einer Gerinnungsaktivierung sowie das Risiko einer Überschwemmung des Patienten mit Zytokinen, Endotoxinen und anderen biologisch aktiven Substanzen. Das Blut wird deshalb im Aufarbeitungsgerät filtriert und zentrifugiert. Hierbei werden insbesondere folgende Bestandteile entfernt:
- aktivierte und nicht aktivierte Gerinnungsfaktoren

- Komplementfaktoren

- freies Hämoglobin

- Thrombozyten

- Leukozyten (je nach System bis zu 99\% Reduktion)

- Heparin

- Antibiotika

- Fett (je nach System bis zu 99,8\%)

- Entzündungsmediatoren

Im Gegenzug werden die Erythrozyten angereichert. 


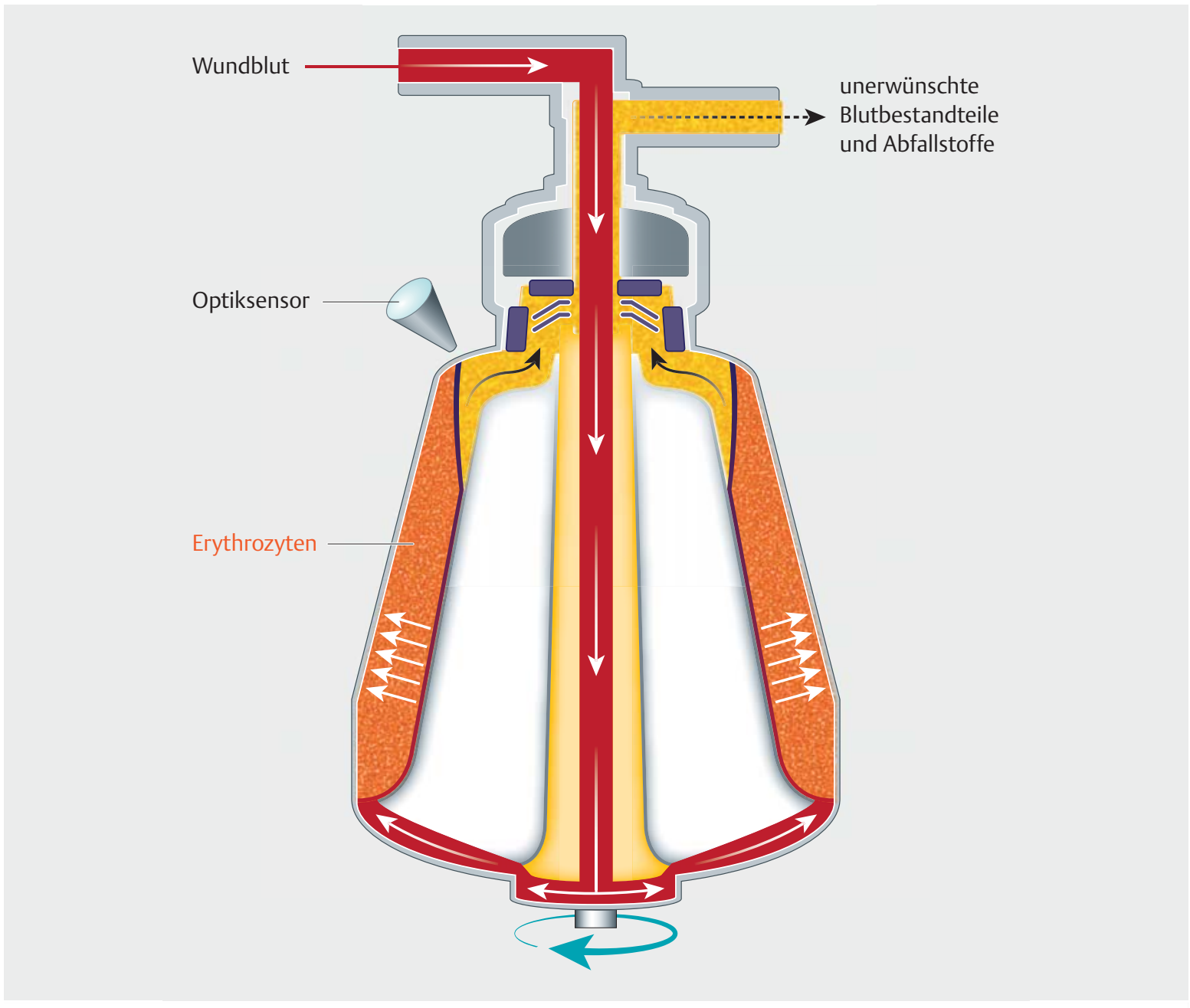

- Abb. 1 Latham-Glocke zur Blutkomponententrennung (Fa. Haemonetics) (Quelle: Andrea Schnitzler/Thieme Gruppe).

- Cave

Der Fettgehalt im retransfundierten Blut muss so weit wie möglich reduziert werden, um der Gefahr einer Fettembolie vorzubeugen.

Zur wirksamen Entfernung des Fetts stehen spezielle Filter mit einer Porengröße von $40 \mu \mathrm{m}$ sowie spezifische Waschprogramme zur Verfügung.

\section{Systeme}

Bei der MAT wird das Wundblut mittels eines sterilen Saugers und einer Vakuumpumpe aus dem Operationsgebiet gesaugt und in einem Reservoir gesammelt. Um das Koagulieren des Blutes im Schlauchsystem und im Auffangbehälter zu verhindern, wird dem Blut eine Heparinlösung beigemischt.

Eine Besonderheit stellt die Anwendung der MAT bei Vorliegen einer heparininduzierten Thrombozytopenie (HIT) dar. Zur Antikoagulation stehen mehrere Alternativen zur Verfügung. Der Einsatz von alternativen Gerinnungshemmern im MAT-System ist von den Herstellern der ma- schinellen Autotransfusion nicht als Zulassung beantragt. Er ist aber als vertretbarer Off-Label-Use im Notfall ein sicheres Verfahren, wenn die richtige Dosierung gewählt wird. Zur Verfügung stehen prinzipiell [1,2]:

- direkte Thrombininhibitoren (z. B. Argatroban)

- Hemmung der Faktor-Xa-Aktivität (z. B. Danaparoid)

- Citrat (2\%ige oder 4\%ige Citrat-Lösung)

- CPDA1 (Citrat-Phosphat-Dextrose-Adenin-Lösung)

Nach Spülung des MAT-Systems mit 100-200 ml der jeweiligen Lösung wird der Zufluss der Antikoagulationslösung in einem Verhältnis zu Wundblut von 1:5 reguliert.

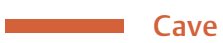

Eine zusätzliche Antikoagulation bei Aufbereitung von bereits defibriniertem postoperativem Drainageblut ist in der Regel nicht notwendig.

Für die Aufarbeitung des gewonnenen Blutes stehen 3 Systeme zur Verfügung. Alle Systeme garantieren folgende Sollwerte des hergestellten Erythrozytenkonzentrats: 


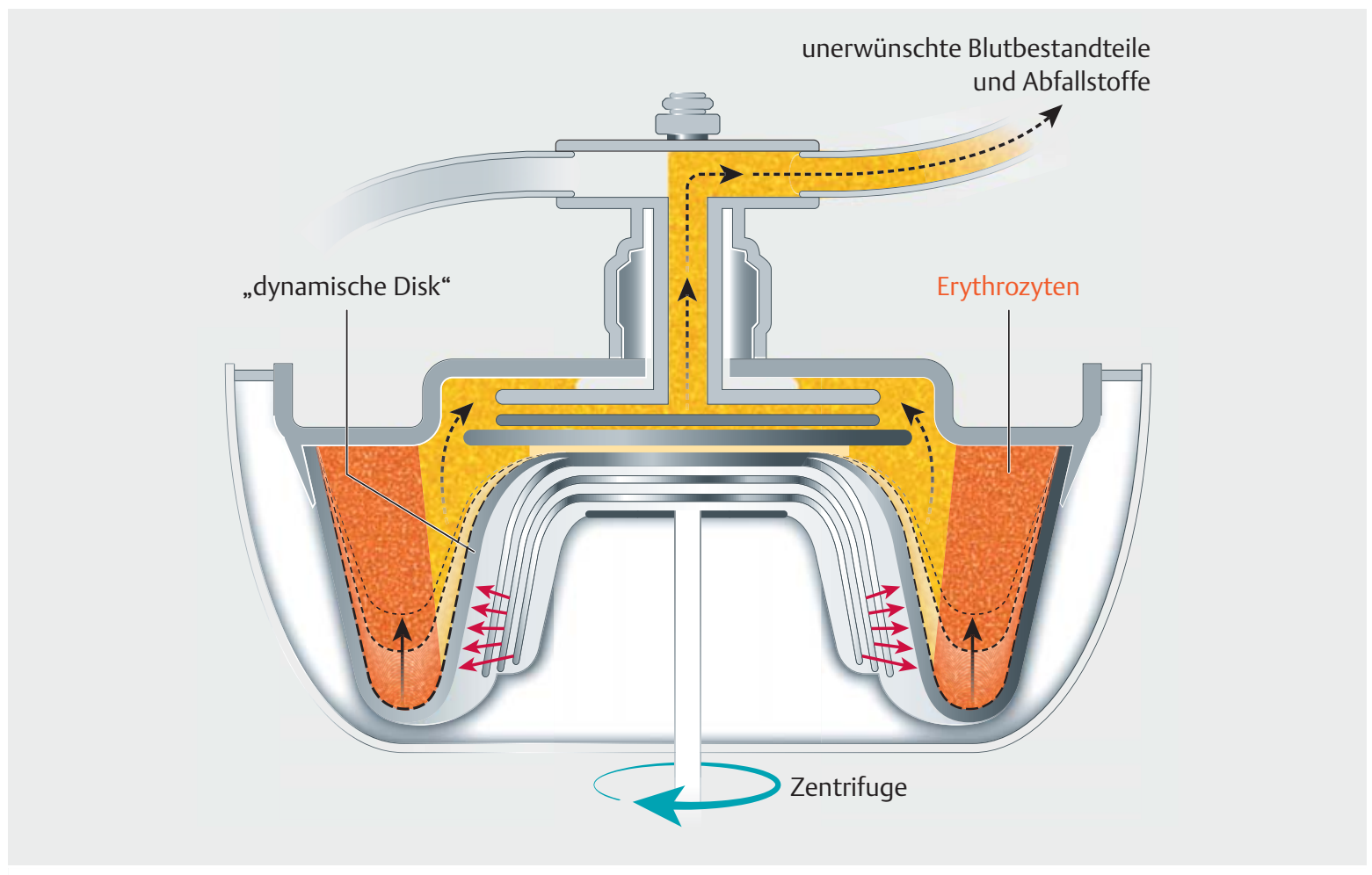

- Abb. 2 Dynamic Disk System (OrthoPAT ${ }^{\circledR}$, Fa. Haemonetics) (Quelle: Andrea Schnitzler/Thieme Gruppe).

- Hämatokrit >50\%

- Erythrozyten-Ausbeute $>80 \%$

- Plasma-/Eiweiß-Elimination $>90 \%$

\section{Latham-Glocke}

Bei der Latham-Glocke handelt es sich um ein diskontinuierliches System, das aus einem inneren, stationären und einem äußeren, rotierenden Teil aufgebaut ist ( $\bullet$ Abb. 1). Ein Teil des im Reservoir gesammelten Blutes wird zunächst in ein glockenähnliches Gefäß gepumpt. Das Blut wird dann in dieser Glocke bei ca. $6000 \mathrm{U} / \mathrm{min}$ zentrifugiert, wobei sich die Erythrozyten an der äußeren Gefäßwand sammeln. Unerwünschte Blutbestandteile und andere Stoffe wandern aufgrund ihres geringeren Gewichtes aufwärts und fließen ab. Bei Überschreitung einer bestimmten Füllhöhe des Zellsediments wird der Waschvorgang mit physiologischer Kochsalzlösung eingeleitet.

Die Glocke kann beliebig oft gefüllt werden. Zu beachten ist hierbei unbedingt die angegebene Mindestfüllmenge der Glocke, um ein Vorbeifließen der Waschlösung am Zellsediment zu verhindern. Dies würde zu einer deutlich schlechteren Auswaschrate und -qualität führen.

\section{Dynamic Disk System}

Auch das Dynamic Disk System ist ein diskontinuierliches System - d.h. mit einer getrennten Füll-, Wasch- und Leerphase - und ähnelt in seinem Funktionsprinzip der Latham-Glocke. Im Gegensatz zur Latham-Glocke ist die
Zentrifugenkammer bei diesem System jedoch nicht starr, sondern eine „dynamische Disk“ ( $\triangleright$ Abb. 2). Im Inneren einer rotierenden Scheibe befindet sich eine Silikonmembran, die sich volumenabhängig in ihrer Form anpasst, sodass keine bestimmte Füllhöhe nötig ist. Pro Zyklus können maximal $100 \mathrm{ml}$ Blut umgesetzt werden. Dadurch eignet sich dieses System besonders bei langsamen Blutverlusten, wie sie bei postoperativen Drainagen nach orthopädischen Eingriffen auftreten.

\section{Elutriatorprinzip (C. A. T. S.; Continuous Autotrans- fusion System)}

Im Gegensatz zu den zuvor beschriebenen Systemen laufen bei diesem die Aufbereitungsphasen kontinuierlich nach dem Elutriatorprinzip (wie bei Apheresen) ab ( $\triangleright$ Abb. 3). Nach Füllung des Systems mit einer initialen Blutmenge von $30 \mathrm{ml}$ laufen bei weiterem Zufluss von Wundblut die Waschung und der Verwurf getrennt ab. Der große Vorteil des kontinuierlichen Systems liegt darin, dass aufgrund des parallel ablaufenden Befüllens, Waschens und Retransfundierens keine Mindestfüllmenge erforderlich ist. Somit können auch kleine Mengen Konzentrat gewonnen werden.

\section{Merke}

Ein weiterer Vorteil dieses Systems betrifft die Fettelimination, die bei 99,8\% liegt, während die diskontinuierlichen Systeme nur Eliminationsraten von $69-93 \%$ erreichen. 


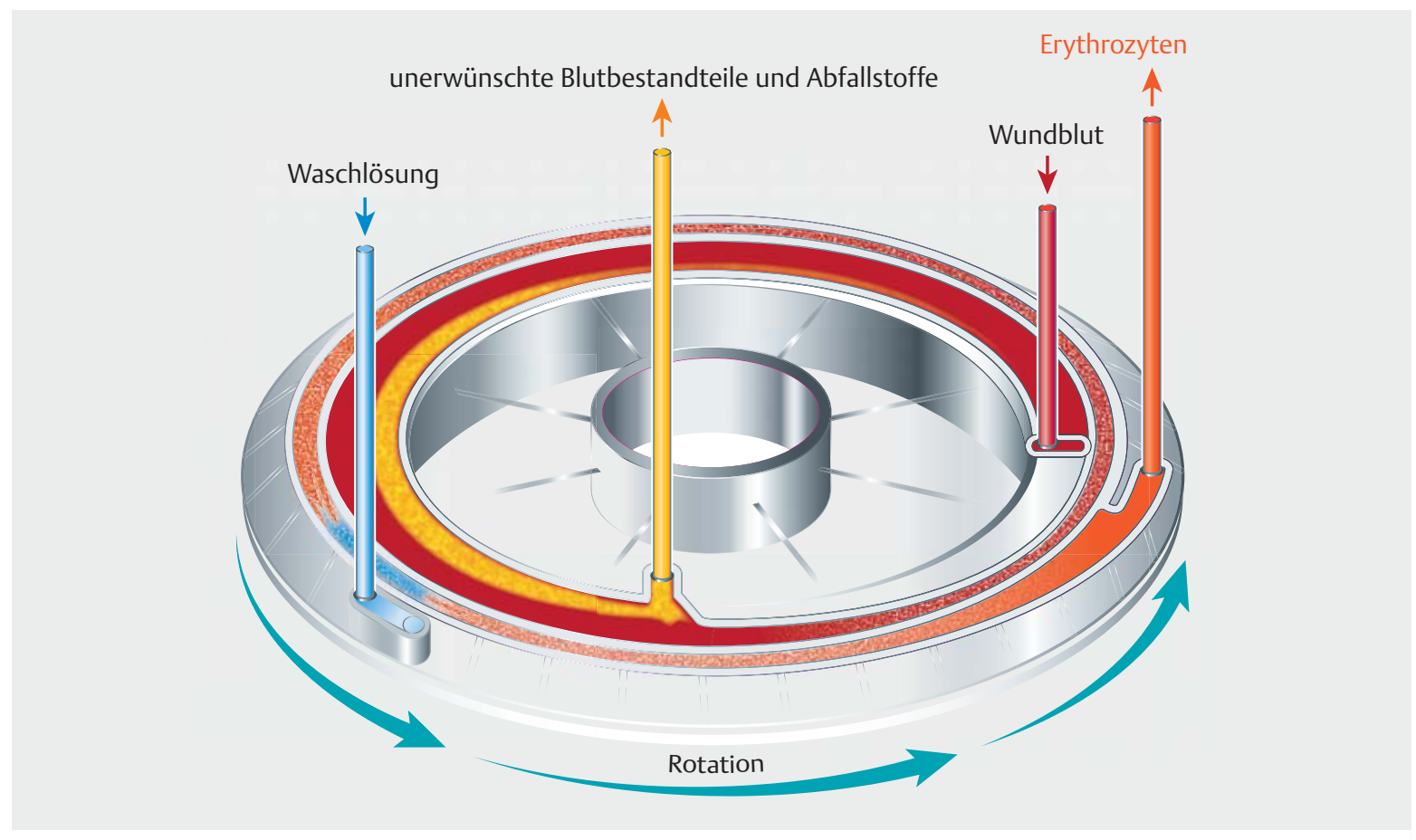

- Abb. 3 Kontinuierliches System nach dem Elutriatorprinzip der Fa. Fresenius Kabi (Quelle: Andrea Schnitzler/Thieme Gruppe).

\section{Vorteile, Indikationen und Kontraindikationen}

Wichtige Vorteile sind die Reduktion der Anzahl fremdbluttransfundierter Patienten und der Fremdbluttransfusionen pro Patient, die hohe Blutqualität und Sicherheit des Blutproduktes sowie die schnelle Verfügbarkeit (s. „Info - Wichtigste Vorteile der MAT“). Die Indikationen und Kontraindikationen der MAT zeigt $•$ Tab. 1.

\section{INFO}

\section{Wichtigste Vorteile der MAT}

- Reduktion des Fremdblutverbrauchs

- Vermeidung von potenziellen Transfusionsrisiken

- Sicherstellung einer hohen Blutqualität

- Vermeidung von lagerungsbedingten Schäden an den Erythrozyten

- hohe Sicherheit des Blutproduktes

- schnelle Verfügbarkeit

- Vermeidung von Fehltransfusionen und Übertragung von Viren und Bakterien

- Ermöglichung von Operationen auch bei Patienten mit komplexen Blutgruppen/Antikörpern

\section{Merke}

Virale systemische Infektionen (z. B. HIV-Infektion, Hepatitis) sind möglich und keine Kontraindikation für den Einsatz der MAT.

\section{Einsatzgebiete}

Die MAT findet hauptsächlich während und nach einer Operation Anwendung. Während der Operation wird Wundblut aus dem Operationsgebiet oder Drainagen gesammelt, aufbereitet und während der Operation retransfundiert. Das maximale Zeitfenster für die Retransfusion beträgt hierbei 6 Stunden. Nach der Operation wird die MAT zum „Recycling“ von ausgetretenem Drainageblut - insbesondere nach orthopädischen Eingriffen oder bei herzchirurgischen Patienten mit postoperativer Nachblutung und Re-Thorax - genutzt, das dem Patienten sofort nach Reinigung wieder zurückgegeben wird.

Die MAT wird hauptsächlich bei Operationen eingesetzt, bei denen Blutverluste $>500 \mathrm{ml}$ zu erwarten sind. Hierzu gehören insbesondere Herz- und Gefäßoperationen, Abdominal- und Transplantationschirurgie, orthopädische und unfallchirurgische Operationen und mit Einschränkungen Tumoroperationen und geburtshilfliche Eingriffe. 
- Tab. 1 Indikationen und Kontraindikationen einer maschinellen Autotransfusion (MAT).

\section{Indikationen}

- erwarteter Blutverlust (>500 ml)

- niedriger Hb-Wert und/oder hohes Blutungsrisiko

- spezifische Antikörper/seltene Blutgruppe

- Ablehnung von Fremdblutprodukten (z. B. Zeugen Jehovas, jedoch als individuelle Gewissensentscheidung)

\section{Kontraindikationen}

- bakterielle Kontamination des Wund-/OP-Gebiets

- Verunreinigung mit Kot oder Eiter

- Tumorchirurgie (bedingt)

- akute systemische Infektionen (z. B. Sepsis)

- zeitgleicher Einsatz von desinfizierenden Spüllösungen im OP-Gebiet (z. B. Lavasept, Betaisodona, Betaseptic ...)

- Aspiration von amniotischer Flüssigkeit oder Magensaft (proteolytische Enzyme dieser Flüssigkeiten könnten zu Störungen der Blutgerinnung führen)

- Aspiration von Antibiotika, die nicht für den intravenösen Gebrauch zugelassen sind (bei orthopädischen Operationen aus Zementzusatz; Gefahr ernsthafter Reaktionen [Hypotonie, Schock])

\section{FALLBEISPIEL}

\section{Herzchirurgie}

Ein 66-jähriger Patient mit einer Aortenklappenstenose, Mitralklappeninsuffizienz und einer 3-GefäßKrankheit wurde mit einem Hb-Wert von $11,3 \mathrm{~g} / \mathrm{dl}$ zum Aortenklappenersatz und zur Anlage eines aortokoronaren Bypasses aufgenommen. Die weitere Diagnostik in der Anämieambulanz konnte einen Eisenmangel ausschließen. Während der OP wurde eine MAT zur Transfusion von autologem Blut verwendet. Es wurden insgesamt $800 \mathrm{ml}$ Wundblut, versetzt mit $500 \mathrm{ml}$ heparinisierter Kochsalzlösung, aufgefangen. Davon wurden $200 \mathrm{ml}$ aufbereitetes Blut retransfundiert. Perioperativ wurden keine zusätzlichen allogenen Blutprodukte benötigt. Der Patient konnte 2 Wochen nach seiner OP mit einem Hb-Wert von $11,8 \mathrm{~g} / \mathrm{dl}$ entlassen werden.

\section{Herz- und Gefäßchirurgie}

\section{Merke}

Eines der wichtigsten Anwendungsgebiete für die MAT ist die Herz- und Gefäßchirurgie.

Die Evidenz für den Einsatz der MAT bei herzchirurgischen Eingriffen - insbesondere bei Einsatz der Herz-Lungen-Maschine - kann gemäß aktueller Metaanalysen und Leitlinien als hoch angesetzt werden [3,4]. Bei Eingriffen am Herzen und großen Gefäßen kommt es sehr häufig zu größeren Blutverlusten, die einen hohen Fremdbluteinsatz nötig machen. Die MAT hilft, diesen Fremdblutbedarf und die damit verbundenen Risiken auf ein Minimum zu senken.

Eine aktuelle Metaanalyse von randomisierten, kontrollierten Studien ergab eine Reduktion des Fremdblutbedarfs um 29\% bei Anwendung der MAT bei herzchirurgischen Eingriffen [5]. Neben der signifikanten Reduktion von Fremdblut konnten darüber hinaus geringere Komplikationsraten, weniger postoperative Infektionen und verminderte kognitive Defizite durch Vermeidung von Fremdblut beobachtet werden $[4,6]$.

Auch bei großen Gefäßoperationen kann der Einsatz der MAT den Verbrauch von Fremdblut relevant senken, was eine Metaanalyse von 4 randomisierten, kontrollierten Studien von Aortenaneurysmaoperationen bestätigt [7].

\section{FALLBEISPIEL}

\section{Not-OP bei Verdacht auf HIT II}

Ein 71-jähriger Patient mit Verdacht auf HIT II musste sich nach einer Herzklappenersatz-OP einer notfallmäßigen Re-OP unterziehen. Der Patient wurde mit Argatroban per Perfusor antikoaguliert. Der Hb-Wert lag vor der Not-OP bei 12,2 g/dl. Das MAT-System wurde vor dem Einsatz mit 50 mg Argatroban pro $1000 \mathrm{ml} \mathrm{NaCl}$ durchgespült und diese Lösung wurde im Verhältnis 1:5 dem Wundblut beigesetzt [1, 2]. Intraoperativ kam es zu einem Blutverlust von $800 \mathrm{ml}$, von denen $430 \mathrm{ml}$ retransfundiert wurden. Der HbWert lag postoperativ bei 11,5 g/dl. Der Patient konnte 15 Tage nach der Not-OP mit einem Hb-Wert von $12,7 \mathrm{~g} / \mathrm{dl}$ entlassen werden.

\section{Orthopädische Eingriffe}

Insbesondere bei endoprothetischen Eingriffen am Knie oder an der Hüfte, bei Wechseloperationen sowie größeren Wirbelsäulenoperationen wird die MAT regelmäßig eingesetzt. Auch postoperativ hat sich die MAT zur Retransfusion des austretenden Wunddrainageblutes bewährt. Eine randomisierte, kontrollierte Studie zeigte, dass sich bei Knieendoprotheseoperationen die Fremdbluttransfusionsrate bei Einsatz der MAT drastisch verringerte (von 80 auf 16\%) [8]. Eine Metaanalyse randomisierter, kontrollierter Studien mit 5631 Patienten konnte die Reduktion von Fremdblutprodukten bei Einsatz der MAT bei Hüft- und Knie-Arthroplastiken erneut bestätigen [9]. 


\section{Tumorchirurgie}

Die Anwendung der MAT in der Tumorchirurgie wird kontrovers diskutiert. Auf der einen Seite birgt die Tumorchirurgie hinsichtlich des Einsatzes von Fremdblut eine spezielle Problematik: Denn aufgrund immunsupprimierender Therapien verlaufen die Reaktionen auf Fremdblut bei diesen Patienten häufig intensiver. Außerdem wurde eine höhere Rezidiv- und Metastasierungsrate bei Tumorpatienten, die Fremdblut erhalten haben, beobachtet.

Auf der anderen Seite erfordert gerade die Tumorchirurgie einen hohen Fremdbluteinsatz. Ungefähr ein Viertel aller Blutkonserven wird in Deutschland bei Tumorpatienten transfundiert. Die Tumorchirurgie ist eigentlich eine Kontraindikation zur MAT, da potenzielle Tumorzellen aus dem Operationsgebiet via aufbereitetem MAT-Blut wieder in die Blutzirkulation zurückgelangen könnten. Der Nachweis von vitalen Tumorzellen (bis $10^{7}$ ) im Wundblut stellt hierbei das größte Problem dar. Allerdings deuten neuere Arbeiten darauf hin, dass Tumorzellen im Wundblut kein Metastasierungspotenzial besitzen [10].

Merke

Zukünftig könnte der Einsatz der MAT auch bei Tumoroperationen eine wirksame Alternative werden.

Zur Inaktivierung von potenziell zirkulierenden Tumorzellen stehen 2 Methoden zur Verfügung:

\section{Bestrahlung}

Eine 50-Gy-Blutbestrahlung des MAT-Bluts kann eine Reduktion an teilungsfähigen Zellen von 12 log-Stufen erreichen. Sie ist somit sehr wahrscheinlich ausreichend, alle Tumorzellen in ihrer Teilungsfähigkeit zu behindern. Die kernlosen Erythrozyten werden bei dieser Strahlendosis nicht beeinträchtigt, sodass weder eine Hämolyse, ein Kaliumaustritt noch eine Veränderung ihrer Funktion (2,3-Diphosphoglycerat) beobachtet werden konnte.

Eine Bestrahlung des gewonnenen Erythrozytenkonzentrats mit 50 Gy kann also sowohl hinsichtlich zuverlässiger Tumorzellelimination als auch Blutqualität als sicher angesehen werden. Die Herausforderung liegt allein in der Logistik und Organisation der Bestrahlung. Denn im Rahmen einer herstellungserlaubnisfreien Anwendung muss die Entnahme, Bestrahlung und Retransfusion vom selben Arzt durchgeführt werden.

Bei Operationen, die nicht im Tumorgebiet erfolgen, ist eine Bestrahlung nicht notwendig.

\section{Leukozytendepletionsfilter}

Verschiedene Studien und systematische Übersichtsarbeiten haben interessanterweise die Effektivität von Leukozytendepletionsfiltern (LDF) bei der Reduktion der Tumorzellen im Rahmen der Retransfusion bestätigt [11, 12]. Die aktuelle Hämotherapierichtlinie greift diese Op- tion (noch) nicht auf. Somit ist bis zum Vorliegen weiterer klinischer Studien die Anwendung nur im Einzelfall (z. B. Tumorchirurgie bei Nichtverfügbarkeit von allogenen Blutprodukten) indiziert.

\section{FALLBEISPIEL \\ Tumorchirurgie}

Ein 58-jähriger Patient, der der Glaubensgemeinschaft der Zeugen Jehovas angehört, musste sich aufgrund eines Prostatakarzinoms einer radikalen Prostataentfernung unterziehen. Der Hb-Wert betrug bei Aufnahme $15 \mathrm{~g} / \mathrm{dl}$ und es lag kein Eisenmangel vor. Aufgrund des erwarteten Blutverlustes von $>500 \mathrm{ml}$ stimmte der Patient dem Einsatz einer MAT zu. Intraoperativ kam es zu einem Blutverlust von 920 ml. Hiervon konnten 506 ml Konzentrat über einen LDF retransfundiert werden. Einen Tag nach der OP lag der HbWert bei $12,0 \mathrm{~g} / \mathrm{dl}$. Nach 11 Tagen konnte der Patient mit einem Hb-Wert von 13,8 g/dl entlassen werden.

\section{Geburtshilfe}

Der Einsatz der MAT in der Geburtshilfe lässt ebenso Raum für Diskussionen. Auf der einen Seite ist der Einsatz der MAT bei postpartaler Blutung aufgrund der großen, teils lebensbedrohlichen Blutverluste zur Vermeidung von Fremdbluttransfusionen sinnvoll. Auf der anderen Seite gibt es in der Geburtshilfe besondere Risiken, die vor Anwendung einer MAT zu beachten sind. Zum einen besteht durch Kontamination der gesammelten Erythrozyten mit Fruchtwasser die Gefahr der gefürchteten Fruchtwasserembolie, die jedoch mithilfe von LDF stark reduziert werden kann. Zum anderen kann es theoretisch zu einer Alloimmunisierung der Mutter mit fetalen Erythrozyten kommen. Denn diese lassen sich aufgrund der homogenen Sedimentationsdichte nicht von den mütterlichen Erythrozyten abtrennen. Diese Alloimmunisierung kann bei Rh-Inkompatibilitäten in späteren Schwangerschaften zu Problemen führen.

\footnotetext{
Merke

Inzwischen zeigen verschiedene Studien weder eine erhöhte Rate an Fruchtwasserembolien noch ein erhöhtes mütterliches Immunisierungsrisiko durch die MAT $[13,14]$.
}

Insofern könnte die MAT wie auch in der britischen MATLeitlinie [15] vermehrt bei geburtshilflichen Eingriffen indiziert sein. Bislang wird sie in der deutschen Geburtshilfe nur in speziellen Situationen angewendet, z. B.

- atone postpartale Blutung,

- spezielle Antikörper-/Blutgruppensituation,

- spezielle Krankheitsbilder (z. B. Placenta praevia) oder

- nicht vorhandene Alternativmethoden (z.B. Zeugen Jehovas). 


\section{Massivtransfusion}

Bei akuter Massivblutung und bei Polytrauma mit massiven Blutverlusten hat die MAT einen festen Stellenwert und kann lebensrettend sein [16]. Ähnliches gilt für andere Operationen mit hohen Blutverlusten, z. B. bei Lebertransplantationen.

\section{Komplikationen}

Zu den theoretisch möglichen Komplikationen der MAT zählen die Verdünnungskoagulopathie, Infektionen oder eine Aktivierung des Immunsystems.

\section{Verdünnungskoagulopathie}

Bei der MAT werden Gerinnungsfaktoren und Thrombozyten eliminiert. Daher kann es in seltenen Fällen bei großem Volumenersatz und alleinigem Einsatz der MAT zu einer Koagulopathie kommen („salvaged-cell syndrome“). Vor diesem Hintergrund muss bei Massivblutung parallel immer ein adäquates Gerinnungsmanagement erfolgen.

\section{Infektionen}

Das Risiko für eine bakterielle Infektion von Wund- und damit MAT-Blut ist relevant. Laut Hämotherapierichtlinie ist der Verbrauch von MAT-Blut aus diesem Grund auf 6 Stunden begrenzt, um eine Vermehrung von möglichen Bakterien zu limitieren.

Die Abnahme von Blutkulturen aus dem MAT-Konzentrat ist allerdings nicht notwendig.

\section{Cave}

Bei Blut, das während der Operation bakteriell kontaminiert wurde (z. B. durch Stuhl, Pus), ist eine MAT absolut kontraindiziert!

\section{Neue Richtlinie Hämotherapie 2017}

2017 erschien die Gesamtnovelle der Richtlinie zur Gewinnung von Blut und Blutbestandteilen und zur Anwendung von Blutprodukten (Richtlinie Hämotherapie) der Bundesärztekammer [17]. Erstmals wurde das Patient Blood Management (PBM) in die Richtlinie aufgenommen [17]. In der aktuellen Richtlinie wird insbesondere auf die autologen Hämotherapieverfahren, die im Rahmen von PBM zunehmend an Bedeutung gewinnen, Wert gelegt. Die wichtigste Methode der autologen Hämotherapie stellt die MAT dar.

\section{Änderungen bezüglich MAT}

Herstellung und Gabe von MAT-Blut

Die Retransfusion von ungefiltertem bzw. unprozessiertem Blut ist aufgrund der Gefahr einer Gerinnungsaktivierung, Zytokin- und Endotoxineinschwemmung sowie Ein- schwemmung anderer biologisch aktiver Substanzen nicht mehr zulässig.

Eigenblutprodukte sind prinzipiell gemäß Arzneimittelgesetz (AMG) als Arzneimittel eingestuft und die Anwendung bedarf prinzipiell einer Herstellungserlaubnis. Eine Herstellungserlaubnis wiederum ist mit einem relativ hohen administrativen und personellen Aufwand verbunden, bedarf der Qualifikation als „Transfusionsmediziner“ und unterliegt den Regularien der Herstellung von Arzneimitteln. Damit wäre die MAT mit Ausnahme von wenigen Krankenhäusern nicht mehr anwendbar. Unter bestimmten Voraussetzungen ist die MAT allerdings nun herstellungserlaubnisfrei (s. „Rechtliches - Herstellungserlaubnis“) und bedarf nur der Anzeigepflicht bei der zuständigen Landesbehörde (Regierungspräsidium).

\section{RECHTLICHES}

\section{Herstellungserlaubnis}

Solange Eigenblut mittels MAT unter der „unmittelbaren fachlichen Verantwortung des anwendenden Arztes hergestellt wird“, das Präparat unmittelbar am Patienten verbleibt und zwischen Entnahme und Retransfusion kein personeller Wechsel stattfindet, ist keine Herstellungserlaubnis erforderlich ( $§ 13$ Abs. 2b AMG).

Im Falle einer Bestrahlung ist darauf zu achten, dass Entnahme und Bestrahlung vom selben Arzt durchgeführt werden, ansonsten wird eine Herstellungserlaubnis nach § 13 AMG erforderlich.

\section{Aufklärung und Dokumentation}

Gemäß § 13 Abs. 1 S. 2 TFG (Transfusionsgesetz) müssen die Anforderungen an die Aufklärung und Einwilligung für den Empfänger von Blutprodukten beachtet werden. Diese Anforderungen umfassen sowohl die Aufklärung vor der Maßnahme bzw. eine nachträgliche Sicherungsaufklärung des Patienten z. B. bei Notfällen.

Ähnlich wie bei allogenen Transfusionen legt die neue Richtlinie auch bei autologen Verfahren besonderen Wert auf eine detaillierte Dokumentation des verwendeten Blutes. Hierzu gehören

- Patientendaten,

- Zeitangaben,

- Name des verantwortlichen Arztes,

- Typ und Nummer des verwendeten Gerätes sowie Chargennummer des verwendeten Systems,

- Berechnung des gesammelten Volumens,

- Angabe des Hämatokrits und der Menge des retransfundierten Blutes. 


\section{Merke}

Für MAT-Blut kann - im Gegensatz zur präoperativen Eigenbluttherapie - auf den AB0-Test verzichtet werden, wenn die Präparate unmittelbar am Patienten verbleiben und zwischen Entnahme und Retransfusion kein personeller Wechsel stattfindet.

\section{Qualitätssicherung}

Die leitende ärztliche Person muss mindestens vierteljährlich die Produkt- und Prozessqualität anhand der Herstellungsprotokolle überprüfen und bei Abweichungen Korrekturmaßnahmen einleiten.

Neu in der Richtlinie ist zudem die Qualitätssicherung der MAT-Methode bei mindestens 5\% der Anwendungen bzw. mindestens einmal im Monat. Hierbei müssen die Eliminationsraten von Gesamteiweiß oder Albumin Hämatokrit-korrigiert als regelmäßige Qualitätskontrolle bestimmt werden. Für die Post-MAT-Probe können Albumin/Eiweiß und Hämatokrit aus dem hergestellten Konzentrat entnommen werden (z. B. mittels 3-Wege-Hahn am Infusionsschlauch). Für die Prä-MAT-Probe wäre eine Blutabnahme aus dem Sammelbehälter bzw. MAT-Zufuhrschlauch denkbar, was technisch und hygienisch relativ anspruchsvoll ist. Insofern kann die direkte Albuminbzw. Eiweißbestimmung aus dem Patientenblut (z. B. im Rahmen der präoperativen Routineanalytik) eine pragmatisch einfache Alternative darstellen.

Die Berechnung der Elimination des Gesamteiweißes (GE) erfolgt nach folgender Formel:

$\mathrm{GE}-$ Elimination $[\%]=100-\left\{\frac{\mathrm{GE}_{E K} \times\left(\operatorname{Vol}_{\mathrm{EK}} \times\left(1-\frac{\mathrm{Hkt}_{\mathrm{EK}}}{100}\right)\right)}{\mathrm{GE}_{\mathrm{WB}} \times\left(\operatorname{Vol}_{\mathrm{WB}} \times\left(1-\frac{\mathrm{Hk \textrm {t } _ { \mathrm { WB } }}}{100}\right)\right)}\right\} \times 100$

GE: Gesamteiweiß, EK: Erythrozytenkonzentrat, WB: Wundblut, Vol: Volumen, Hkt: Hämatokrit.

Auf der Seite http://www.patientbloodmanagement.de befindet sich eine Eingabemaske zur Berechnung der Elimination des Gesamteiweißes sowie ein Link zum Herunterladen einer Excel-Tabelle (Pfad: Konzept $\rightarrow$ PBM Maßnahmen im Detail $\rightarrow$ Maschinelle Autotransfusion). Als Sollwerte gelten: Hämatokrit $>50 \%$, Eliminationsrate von Gesamteiweiß > 90\% des Ausgangswertes.

\section{Kosteneffizienz}

Hinsichtlich der Kosteneffizienz bestehen erhebliche Unterschiede zwischen den durchgeführten Studien. Die umfassendste Analyse erfolgte durch Klein et al., die durchschnittliche Kosten von 103 \$ bei der Verwendung der MAT beschrieben [18]. Eine andere Studie ermittelte eine Reduktion der Kosten von $225 €$ pro Patient bei Transfusion von autologem Blut [19]. Limitationen vorhandener Studien sind insbesondere unterschiedliche Berechnungen der Kosten durch Unterschiede bei den ein- bezogenen Parametern wie MAT-Geräte, Personalkosten, Lagerkosten für Blutprodukte, Kosten für Geräteanschaffung und -wartung. Somit ist ein objektiver Vergleich kaum möglich.

Merke

Prinzipiell kann durch ein 2-stufiges Verfahren die MAT kostengünstig eingesetzt werden:

1. Sammeln bei erwartetem Blutverlust

2. Aufbereitung erst bei tatsächlichem Blutverlust von $>500 \mathrm{ml}$

\section{Fazit}

Unter den fremdblutsparenden Maßnahmen, die im Rahmen des PBM angewendet werden, kommt der MAT ein besonderer Stellenwert zu. Die breite Anwendbarkeit der MAT bei herz- und gefäßchirurgischen, orthopädischen Eingriffen und - nach Bestrahlung oder Filterung - bei Tumoroperationen deckt alle Bereiche mit hohen Blutverlusten ab. Die Komplikationsrate bei MAT-Anwendung ist sehr gering. Nur wenige Patienten sind vom Einsatz der MAT ausgeschlossen. Aus diesen Gründen trägt die MAT wesentlich zur Qualität der Blutversorgung bei chirurgischen Patienten bei.

\section{Auszeichnungen}

Das Patient-Blood-Management-Konzept erhielt den Lohfert-Preis 2014 (Christoph Lohfert Stiftung), den Humanitarian Award 2015 (Patient Safety Summit), den Deutschen Preis für Patientensicherheit 2016 (Aktionsbündnis Patientensicherheit, APS), den Julius-Springer-Award 2017 (Deutsche Gesellschaft für Chirurgie), den Publikationspreis 2018 (Deutsche Gesellschaft für Thorax-, Herzund Gefäßchirurgie) sowie den MSD-Gesundheitspreis 2018 (3. Platz).

\section{KERNAUSSAGEN}

- Die MAT ist eine effektive Methode, um den Fremdblutbedarf zu reduzieren.

- Oft ist die MAT die einzige Option, um komplexe Operationen durchführen zu können, z. B. bei Patienten mit komplexen Antikörpern, seltenen Blutgruppen und bei Patienten, die aus religiösen Gründen Fremdblutprodukte ablehnen.

- Haupteinsatzgebiete der MAT sind Operationen am Herzen und großen Gefäßen sowie orthopädische Eingriffe, bei denen Blutverluste $>500 \mathrm{ml}$ erwartet werden.

- Die sofortige Wiederverwendung des MAT-Blutes stellt Erythrozyten in uneingeschränkter Funktionsfähigkeit und Vitalität ohne Lagerungsschäden zur Verfügung.

- Systeme, die kontinuierlich arbeiten, sind den diskontinuierlichen Systemen hinsichtlich Blutverwurfmengen und Fettelimination überlegen. 


\section{Interessenkonflikt}

P. M. und K.Z. erhielten finanzielle Förderungen von B. Braun Melsungen, CSL Behring, Fresenius Kabi und Vifor Pharma für eine Investigator-initiierte Studie zur Implementierung des Patient Blood Management Programms in vier Universitätsklinika. P. M. und/oder K. Z. erhielten Förderungen oder Reisekostenunterstützung für Beratungen und Vorträge der folgenden Firmen: Abbott GmbH \& Co KG, AesculapAkademie $\mathrm{GmbH}$, AQAI GmbH, AstellasPharma GmbH, AstraZeneca $\mathrm{GmbH}$, Aventis Pharma GmbH, B. Braun Melsungen AG, Baxter Deutschland GmbH, Biosyn GmbH, Biotest AG, Bristol-Myers Squibb GmbH, CSL Behring GmbH, Dr. F. KöhlerChemie $\mathrm{GmbH}$, Dräger Medical GmbH, Essex Pharma GmbH, Fresenius Kabi GmbH, Fresenius Medical Care, Gambro Hospal GmbH, Gilead, GlaxoSmithKline GmbH, Grünenthal GmbH, Hamilton Medical AG, HCCM Consulting GmbH, Heinen + Löwenstein $\mathrm{GmbH}$, Janssen-Cilag $\mathrm{GmbH}$, med Update $\mathrm{GmbH}$, Medivance EU B. V., MSD Sharp \& Dohme GmbH, Novartis Pharma GmbH, Novo Nordisk Pharma GmbH, P. J. Dahlhausen \& Co. GmbH, Pfizer Pharma GmbH, Pulsion Medical Systems S.E., Siemens Healthcare, Teflex Medical GmbH, Teva GmbH, TopMedMedizintechnik GmbH, Verathon Medical, ViforPharma GmbH.

\section{Autorinnen/Autoren}

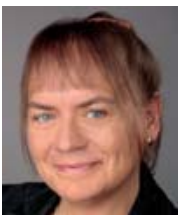

\section{Sabine Westphal}

Dr. rer. nat., wissenschaftliche Mitarbeiterin in der Klinik für Anästhesiologie, Intensivmedizin und Schmerztherapie des Universitätsklinikum Frankfurt am Main. Studium der Biologie in Kiel. Tätigkeiten am Universitätsklinikum Münster, an der Universität Bielefeld und an der Charité Berlin. Seit 2016 beschäftigt am Universitätsklinikum Frankfurt. Wissenschaftliche Schwerpunkte: Patient Blood Management und klinische Studien.

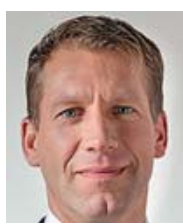

\section{Kai Zacharowski}

Prof. Dr. med. Dr. rer. nat., seit 2009 Direktor der Klinik für Anästhesiologie, Intensivmedizin und Schmerztherapie am Universitätsklinikum Frankfurt am Main. Wissenschaftliche Schwerpunkte: Sepsis, angeborene Immunität, Ischämie-Reperfusions-Syndrom, translationale Medizin, Durchführung klinischer Studien sowie Patient Blood Management. Landesvorsitzender der Deutschen Gesellschaft für Anästhesiologie und Intensivmedizin (DGAI) in Hessen.

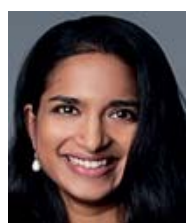

\section{Suma Choorapoikayil}

Dr. rer. nat., seit 2015 wissenschaftliche Mitarbeiterin in der Klinik für Anästhesiologie, Intensivmedizin und Schmerztherapie des Universitätsklinikum Frankfurt am Main. Studium der Biologie mit Promotion im Fachbereich Genetik an der Universität zu Köln. Auslandsaufenthalt am Hubrecht Institute in Utrecht und an der Universität Montpellier. Wissenschaftliche Schwerpunkte: hämatopoetische Stammzellen, Angiogenese und Patient Blood Management.

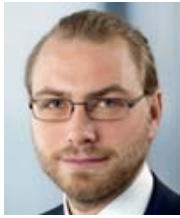

\section{Christoph Füllenbach}

Dr. rer. nat., wissenschaftlicher Mitarbeiter in der Klinik für Anästhesiologie, Intensivmedizin und Schmerztherapie des Universitätsklinikum Frankfurt am Main. Studium der Geologie mit Promotion an der Johannes Gutenberg-Universität Mainz. Seit 2016 beschäftigt am Universitätsklinikum Frankfurt. Wissenschaftliche Schwerpunkte: Anämie und Patient Blood Management.

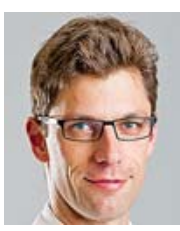

\section{Patrick Meybohm}

Prof. Dr. med., stellvertretender Direktor der Klinik für Anästhesiologie, Intensivmedizin und Schmerztherapie des Universitätsklinikum Frankfurt am Main. Medizinstudium und Promotion an der Georg-August-Universität Göttingen. 2009 Facharzt für Anästhesiologie am Universitätsklinikum Schleswig-Holstein, Campus Kiel. Seit 2012 am Universitätsklinikum Frankfurt tätig. Schwerpunkte: Intensivmedizin, perioperatives Outcome und Patient Blood Management.

Korrespondenzadresse

Univ.-Prof. Dr. med. Patrick Meybohm

Klinik für Anästhesiologie, Intensivmedizin und Schmerztherapie

Universitätsklinikum Frankfurt am Main

Theodor-Stern-Kai 7

60590 Frankfurt am Main

patrick.meybohm@kgu.de

\section{Wissenschaftlich verantwortlich} gemäß Zertifizierungsbestimmungen

Wissenschaftlich verantwortlich gemäß Zertifizierungsbestimmungen für diesen Beitrag ist Prof. Dr. med. Patrick Meybohm, Frankfurt am Main.

\section{Literatur}

[1] Hönemann C. Patient mit HIT/CRP 12: Fallszenario zum Thema - Leitlinien und Empfehlungen im Praxistest. Anästh Intensivmed 2012; 53: S465-507

[2] IAKH. Cell Saver bei HIT. Meldeauswertung des IAKH-Fehlerregisters [Fall-ID: CM-29283-2016] 2016. Im Internet: http:// www.iakh.de/files/iakh/public/register/CM-29283-2016-CellSaver\%20bei\%20HIT.pdf; Stand: 15.01.2019

[3] Ferraris VA, Ferraris SP, Saha SP et al. Perioperative blood transfusion and blood conservation in cardiac surgery. The Society of Thoracic Surgeons and The Society of Cardiovascular Anesthesiologists clinical practice guideline. Ann Thorac Surg 2007; 83: S27-S86. doi:10.1016/j.athoracsur.2007.02.099

[4] Wang G, Bainbridge D, Martin J et al. The efficacy of an intraoperative cell saver during cardiac surgery. A meta-analysis of randomized trials. Anesth Analg 2009; 109: 320-330. doi:10.1213/ane.0b013e3181aa084c

[5] Meybohm P, Choorapoikayil S, Wessels A et al. Washed cell salvage in surgical patients. A review and meta-analysis of prospective randomized trials under PRISMA. Medicine (Baltimore) 2016; 95: e4490. doi:10.1097/MD.0000000000004490 
[6] Hansen E, Seyfried T. Maschinelle Autotransfusion. Anaesthesist 2011; 60: 381-389; quiz 390. doi:10.1007/s00101-011$1865-4$

[7] Takagi H, Sekino S, Kato T et al. Intraoperative autotransfusion in abdominal aortic aneurysm surgery. Meta-analysis of randomized controlled trials. Arch Surg 2007; 142: 1098-1101. doi:10.1001/archsurg. 142.11.1098

[8] Shenolikar A, Wareham K, Newington D et al. Cell salvage auto transfusion in total knee replacement surgery. Transfus Med 1997; 7: 277-280

[9] van Bodegom-Vos L, Voorn VM, So-Osman C et al. Cell salvage in hip and knee arthroplasty. A meta-analysis of randomized controlled trials. J Bone Joint Surg Am 2015; 97: 1012-1021. doi:10.2106/JBJS.N.00315

[10] Kumar N, Zaw AS, Kantharajanna SB et al. Metastatic efficiency of tumour cells can be impaired by intraoperative cell salvage process. Truth or conjecture? Transfus Med 2017; 27 (Suppl. 5): 327-334. doi:10.1111/tme.12453

[11] Mei K, Du L, Yan M et al. Modified leukocyte filter removes tumor cells from the salvaged blood. PLoS One 2015; 10: e0130864. doi:10.1371/journal.pone.0130864

[12] Kumar N, Chen Y, Zaw AS et al. Use of intraoperative cell-salvage for autologous blood transfusions in metastatic spine tumour surgery. A systematic review. Lancet Oncol 2014; 15: e33-e41. doi:10.1016/S1470-2045(13)70245-6

[13] Allam J, Cox M, Yentis SM. Cell salvage in obstetrics. Int J Obstet Anesth 2008; 17: 37-45. doi:10.1016/j.ijoa.2007.08.001

[14] Sullivan I, Faulds J, Ralph C. Contamination of salvaged maternal blood by amniotic fluid and fetal red cells during elective Caesarean section. Br J Anaesth 2008; 101: 225-229. doi:10.1093/bja/aen135
[15] Klein AA, Bailey CR, Charlton AJ et al. Association of Anaesthetists guidelines: cell salvage for peri-operative blood conservation 2018. Anaesthesia 2018; 73: 1141-1150

[16] AWMF. S3-Leitlinie Polytrauma/Schwerverletzten-Behandlung AWMF-Register-Nr. 012/019. Im Internet: http://www.awmf. org/uploads/tx_szleitlinien/012-019k_S3_Polytrauma_ Schwerverletzten-Behandlung_2017-03.pdf; Stand: 15.01.2019

[17] Bundesärztekammer. Richtlinie zur Gewinnung von Blut und Blutbestandteilen und zur Anwendung von Blutprodukten (Richtlinie Hämotherapie), Gesamtnovelle 2017. Im Internet: http://www.bundesaerztekammer.de/fileadmin/user_upload/ downloads/pdf-Ordner/MuE/Richtlinie_Haemotherapie_2017. pdf; Stand: 15.01.2019

[18] Klein AA, Nashef SAM, Sharples L et al. A randomized controlled trial of cell salvage in routine cardiac surgery. Anesth Analg 2008; 107: 1487-1495. doi:10.1213/ane.0b013 e3181831e54

[19] Savvidou C, Chatziioannou SN, Pilichou A et al. Efficacy and cost-effectiveness of cell saving blood autotransfusion in adult lumbar fusion. Transfus Med 2009; 19: 202-206. doi:10.1111/ j.1365-3148.2009.00929.x

Bibliografie

DOI https://doi.org/10.1055/a-0593-4377

Anästhesiol Intensivmed Notfallmed Schmerzther 2019; 54: 170-181 @ Georg Thieme Verlag KG Stuttgart · New York ISSN 0939-2661 


\section{Punkte sammeln auf CME.thieme.de}

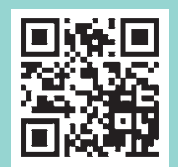

Diese Fortbildungseinheit ist in der Regel 12 Monate online für die Teilnahme verfügbar.

Den genauen Einsendeschluss finden Sie unter https://eref.thieme.de/CXAQ1 KL.

Sollten Sie Fragen zur Online-Teilnahme haben, finden Sie unter https://cme.thieme.de/hilfe

eine ausführliche Anleitung. Wir wünschen viel Erfolg beim Beantworten

der Fragen!

Unter https://eref.thieme.de/CXAQ1KL oder über den QR-Code kommen Sie direkt zur Startseite des Wissenstests.

VNR 2760512019156641225

\section{Frage 1}

\section{Welche Aussage ist richtig?}

A Bei erwarteten hohen Blutverlusten ist die maschinelle Autotransfusion (MAT) ungeeignet.

B Bestehende Infektionen des Patienten sind für die MAT irrelevant.

C Eine MAT ist der Retransfusion von ungewaschenem Wundblut in Sicherheit und Effizienz überlegen.

D Die Latham-Glocke wird mit Fremdblut gefüllt.

E Blut, das nicht benötigt wird, wird für den nächsten Patienten aufbewahrt.

\section{Frage 2}

\section{Welche Aussage ist falsch?}

A Die MAT reduziert das theoretische fremdbluttransfusionsbedingte Risiko einer Infektion mit HIV.

B Die Anwendung der MAT ist besonders bei Patienten mit seltener Blutgruppe indiziert.

C Die MAT filtert zirkulierende Bakterien aus dem Blut.

D Die MAT stellt für die Zeugen Jehovas oftmals die einzige Möglichkeit dar, sich großen Operationen zu unterziehen.

E Die Erythrozytenqualität bei Verwendung der MAT ist wesentlich höher als in gelagerten Fremdblutkonzentraten.

\section{Frage 3}

Bei welcher bestehenden Infektion darf die MAT nicht verwendet werden?
A HIV (humanes Immundefizienz-Virus)
B HCV (Hepatitis-C-Virus)
C Peritonitis
D HSV (Herpes-simplex-Virus)
E Influenza

\section{Frage 4}

Welche Aussage ist richtig?

A Bei Patienten mit Leukämie ist eine MAT nur nach vorheriger Bestrahlung des Blutes möglich.

B Bei Operationen, die nicht im Tumorgebiet erfolgen, ist eine Bestrahlung nicht notwendig.

C Vom bestrahlten MAT-Blut und vom Patientenblut ist vor Retransfusion in jedem Fall ein Blutgruppentest durchzuführen.

D Anstelle einer Bestrahlung kann auch eine Zentrifugation des Blutes erfolgen.

E Bei Patienten, bei denen der Tumor bereits Metastasen gebildet hat, ist keine Bestrahlung notwendig.

\section{Frage 5}

Was ist keine Neuerung der neuen Richtlinie Hämotherapie von 2017 ?

A Die Retransfusion von nicht gewaschenem bzw. nicht prozessiertem Blut ist nicht mehr zulässig.

B Solange Eigenblut unter der unmittelbaren fachlichen Verantwortung des anwendenden Arztes hergestellt wird, ist keine Herstellungserlaubnis erforderlich.

C Die neue Richtlinie legt Wert auf eine detaillierte Dokumentation der MAT.

D Es ist eine monatliche Qualitätssicherung durchzuführen.

E Entnahme, Bestrahlung und Retransfusion können von verschiedenen Ärzten durchgeführt werden.

\section{- Weitere Fragen auf der folgenden Seite...}




\title{
Punkte sammeln auf CME.thieme.de
}

\author{
Fortsetzung ...
}

\section{Frage 6}

Welche Aussage zur MAT-Verwendung in der Geburtshilfe ist richtig?

A Hinsichtlich der Verwendung der MAT in der Geburtshilfe gibt es keinerlei Einschränkungen.

B Bei Verwendung der MAT muss jedes Blutkonzentrat vor Retransfusion bestrahlt werden.

C Das Risiko einer Alloimmunisierung der Mutter ist bei Verwendung der MAT sehr hoch.

D Bei der MAT kann eine bestehende HIV-Infektion der Mutter auf das Kind übertragen werden.

E Leukozytendepletionsfilter reduzieren effektiv das Risiko einer Fruchtwasserembolie.

\section{Frage 7}

Was ist keine mögliche Komplikation der MAT?

A Fruchtwasserembolie bei Einsatz im Rahmen einer atonen postpartalen Blutung

B Verdünnungskoagulopathie

C Aktivierung des Immunsystems

D transfusionsassoziierte akute Lungeninsuffizienz (TRALI)

E bakterielle Kontamination

\section{Frage 8}

Welche Aussage ist richtig?

A Die Latham-Glocke ist allen anderen Systemen überlegen.

B Der Leukozytendepletionsfilter reduziert die Leukozyten um bis zu $99 \%$.

C Bei den mit der MAT hergestellten Erythrozytenkonzentraten wird ein möglichst niedriger Hämatokrit $<40 \%$ angestrebt.

D Beim Dynamic Disk System können bis zu $1000 \mathrm{ml}$ Blut pro Zyklus umgesetzt werden, sodass sich dieses System insbesondere bei Operationen mit erwarteten großen Blutverlusten eignet.

E Beim Continuous Autotransfusion System (C. A. T. S.) liegt die Eliminationsrate für Fett bei ca. 50-60\%.

\section{Frage 9}

Ein 75-jähriger Patient mit einer 3-Gefäß-Krankheit und einer Aortenklappenstenose erhält eine aortokoronare Bypassoperation und einen Aortenklappenersatz. Welche Aussage ist richtig?

A Herzchirurgische Eingriffe gehören nicht zu den Haupteinsatzgebieten der MAT.

B Da der erwartete Blutverlust > $500 \mathrm{ml}$ beträgt, ist die Verwendung einer MAT indiziert.

C Bei herzchirurgischen Eingriffen ist eine bestehende HIV-Infektion relevant und führt zum Ausschluss der MAT.

D Die Gefahr einer TRALI ist bei herzchirurgischen Eingriffen bei Verwendung der MAT besonders hoch.

E Gesammeltes Blut kann bei thorax-, herz- und gefäßchirurgischen Eingriffen bis zu 12 Stunden verwendet werden.

\section{Frage 10}

Darf ein Patient mit HIV-Infektion eine MAT bekommen?

A nein

B nur nach Bestrahlung der Blutprobe mit 50 Gy

C nur mit Einsatz von leukozytendepletierenden Filtern

D nur mit begleitender Antibiotikatherapie

E ja 